\title{
AEGIS: GALAXY SPECTRAL ENERGY DISTRIBUTIONS FROM THE X-RAY TO RADIO
}

\author{
N. P. Konidaris, ${ }^{1}$ P. Guhathakurta, ${ }^{1}$ K. Bundy, ${ }^{2}$ A. L. Coil,${ }^{3,4}$ C. J. Conselice, ${ }^{5}$ M. C. Cooper, ${ }^{6}$ P. R. M. Eisenhardt, ${ }^{7}$ \\ J.-S. Huang, ${ }^{8}$ R. J. Ivison, ${ }^{9}$ S. A. Kassin, ${ }^{1}$ E. N. Kirby, ${ }^{1}$ J. M. Lotz, ${ }^{10}$ J. A. Newman,,${ }^{3,11}$ K. G. Noeske, ${ }^{1}$ R. M. Rich, ${ }^{12}$ \\ T. A. Small, ${ }^{13}$ C. N. A. Willmer, ${ }^{4}$ And S. P. Willner ${ }^{8}$ \\ Received 2006 June 7; accepted 2006 September 15; published 2007 April 11
}

\begin{abstract}
The All-Wavelength Extended Groth Strip International Survey (AEGIS) team presents broadband spectral energy distributions (SEDs), from X-ray to radio wavelengths, for 71 galaxies spanning the redshift range 0.55$1.16(\langle z\rangle \sim 0.7)$. Galaxies with secure redshifts are selected from a small $\left(22 \operatorname{arcmin}^{2}\right)$ subsection of the Keck/ DEIMOS Galaxy Redshift Survey in the Extended Groth Strip field that has also been targeted for deep panchromatic imaging by ultraviolet $(120-250 \mathrm{~nm})$, optical $(360-900 \mathrm{~nm})$, optical/near-infrared (440-1600 nm), near-infrared (1200-2200 nm), mid/far-infrared $(3.6-70 \mu \mathrm{m})$, and radio $(6-20 \mathrm{~cm})$. A typical galaxy in our sample is $M_{B}=-19.82$. The ultraviolet to mid-infrared portion of their SEDs are found to be bracketed by two stellaronly model SEDs: (1) an early burst followed by passive evolution and (2) a constant star formation rate since early times. This suggests that few of these galaxies are undergoing major starbursts. Approximately half the galaxies show a mid- to far-infrared excess relative to the model SEDs, consistent with thermal emission from interstellar dust. Two objects have power-law SEDs, indicating that they are dominated by active galactic nuclei; both are detected in X-rays. SEDs, from the ultraviolet to the infrared, follow expected trends: redder SEDs are associated with red $U-B$, early-type morphology, and low [O II] emission, and vice versa for blue SEDs.
\end{abstract}

Subject headings: galaxies: evolution — galaxies: general — infrared: galaxies — radio continuum: galaxies — ultraviolet: galaxies - X-rays: galaxies

\section{INTRODUCTION}

The spectral energy distribution (SED) of a galaxy contains key clues about the dominant physical processes responsible for energy generation. Space- and ground-based telescopes of the new millenium provide the sensitivity and angular resolution to make measurements across the breadth of the electromagnetic spectrum from X-ray to radio wavelengths. Panchromatic SEDs can tell us about the stellar populations within the galaxy from their visible light (and ultraviolet [UV] and near-infrared [IR]) properties (e.g., Bruzual \& Charlot 2003, hereafter $\mathrm{BC} 03$, and references therein), thermal reradiation of starlight in the far-IR by interstellar dust (Dale et al. 2001),

\footnotetext{
${ }^{1}$ UCO Lick Observatory and Department of Astronomy and Astrophysics, University of California, Santa Cruz, CA 95064; npk@ucolick.org, raja@ ucolick.org, kassin@ucolick.org, ekirby@ucolick.org, kai@ucolick.org.

${ }^{2}$ California Institute of Technology, Pasadena, CA 91125; kbundy@astro .caltech.edu.

${ }^{3}$ Hubble Fellow.

${ }^{4}$ Steward Observatory, University of Arizona, Tucson, AZ 85721; acoil@ as.arizona.edu, cnaw@as.arizona.edu.

${ }^{5}$ School of Physics and Astronomy, University of Nottingham, Nottingham NG9 2RD, UK; conselice@ nottingham.ac.uk.

${ }^{6}$ Department of Astronomy, University of California, Berkeley, CA 94720; cooper@astron.berkeley.edu.

${ }^{7}$ Jet Propulsion Laboratory, California Institute of Technology, CA 91109; prme@kromos.jpl.nasa.gov.

${ }^{8}$ Harvard-Smithsonian Center for Astrophysics, Cambridge, MA 02138; jhuang@cfa.harvard.edu, swillner@cfa.harvard.edu.

${ }^{9}$ Astronomy Technology Centre, Royal Observatory, Edinburgh EH9 3HJ, $\mathrm{UK}$; rji@roe.ac.uk.

${ }^{10}$ National Optical Astronomical Observatories, Tucson, AZ 85719; lotz@ noao.edu.

${ }^{11}$ Institute for Nuclear Particle Astrophysics, Lawrence Berkeley National Laboratory, Berkeley, CA 94720; janewman@lbl.gov.

${ }^{12}$ Department of Physics and Astronomy, University of California, Los Angeles, CA 90095; rmr@astro.ucla.edu.

${ }^{13}$ Space Astrophysics, California Institute of Technology, Pasadena, CA 91125; tas@astro.caltech.edu.
}

and X-ray (e.g., Mushotzky et al. 1980) and radio emission from active galactic nuclei (AGNs).

Dale et al. (2001) have studied panchromatic SEDs of nearby galaxies and have developed a semiempirical model for the IR SED of normal star-forming galaxies. Distant galaxy SEDs are important for look-back studies of galaxy formation and evolution. The first such survey was GOODS (Giavalisco et al. 2004). Our project, the All-Wavelength Extended Groth Strip International Survey (AEGIS; Davis et al. 2007, hereafter Paper I), extends the work of GOODS over a larger survey area (in a part of the sky known as the Extended Groth Strip [EGS]), thereby driving down cosmic variance. The cornerstone of AEGIS is the Keck ${ }^{14} /$ DEIMOS DEEP2 Galaxy Redshift Survey (Davis et al. 2003). Precise redshift measurements from this survey allow us to determine the environment of each galaxy (Cooper et al. 2005), a key factor in determining galaxy evolution. Moreover, these DEIMOS spectra allow us to measure line widths and estimate dynamical masses for the galaxies.

This Letter presents SEDs of 71 optically selected galaxies in AEGIS $^{15}$ and is intended to showcase the panchromatic data set. Given the relatively coarse angular resolution of even the state-of-the-art UV and mid/far-IR images in our survey, we limit our study to the global emission from these distant galaxies. The goal of this Letter is to provide clean SEDs for a handful of objects. Throughout this Letter we use a cosmology of $\left(H_{0}, \Omega_{m}, \Omega_{\Lambda}\right)=(70,0.3,0.7)$. Magnitudes are in the $\mathrm{AB}$ system.

\section{THE AEGIS SURVEY AND MTR SUBSAMPLE}

The AEGIS spectroscopic field is a $0.5 \mathrm{deg}^{2}$ field at $\alpha=$ $14^{\mathrm{h}} 17^{\mathrm{m}}, \delta=+52^{\circ} 30^{\prime}$. It is only one of a few regions in the

\footnotetext{
${ }^{14}$ Data presented herein were obtained at the W. M. Keck Observatory, which is operated as a scientific partnership among the California Institute of Technology, the University of California, and NASA. The Observatory was made possible by the generous financial support of the W. M. Keck Foundation.

${ }^{15}$ These data are available at http://aegis.ucolick.org/products.
} 


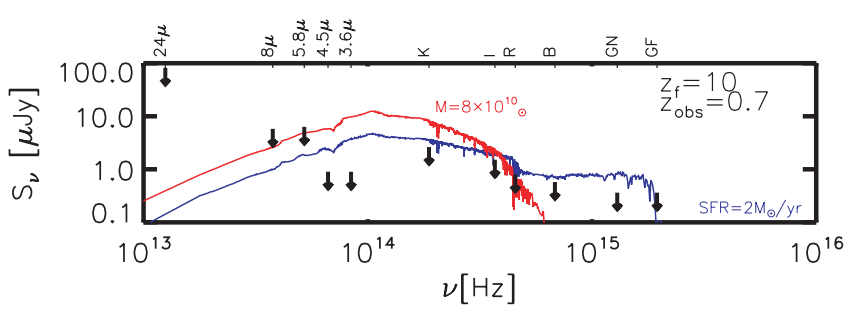

FIG. 1.-AEGIS detection limits (tips of downward arrows) are compared to $\mathrm{BC} 03$ stellar population models in a log flux density $(\mu \mathrm{Jy})$ vs. log frequency $(\mathrm{Hz})$ plot. The red line represents a single early burst $\left(z_{f}=10\right)$ with a stellar mass of $M_{*}=8 \times 10^{10} M_{\odot}$, which has been passively evolved to $z=0.7$. The blue line represents a population that has been forming stars at a constant rate $\left(\dot{M}=2 M_{\odot} \mathrm{yr}^{-1}\right)$. At this rate, the galaxy forms $\sim 1.3 \times 10^{10} M_{\odot}$ in stars between $z_{f}=10$ and $z_{\mathrm{obs}}=0.7$. Neither model considers dust or nebular emission. Model parameters are chosen to yield SEDs that are detectable in the $R$ band.

sky with deep imaging coverage in all major observable wave bands and spectroscopy with a $10 \mathrm{~m}$ class telescope (DEEP2 Keck/DEIMOS redshift survey). Deep imaging exists in Chandra ACIS (X-ray), Galaxy Evolution Explorer (UV), Hubble Space Telescope (HST) Advanced Camera for Surveys (ACS) and NICMOS (optical and near-IR), Canada-France-Hawaii Telescope (CFHT)/MegaCam and CFH12K (multiband optical), Palomar (near-IR), Spitzer (mid- and far-IR), and Very Large Array (6 and $20 \mathrm{~cm}$ radio). Availability of secure spectroscopic redshifts is the primary sample selection criterion. The DEEP2 survey targets galaxies with $R<24.1$ and covers the redshift range $z<1.4$ in the EGS. Detailed selection criteria and effective wavelengths, exposure times, limiting magnitudes, source surface densities, etc., for the AEGIS data sets are presented in Paper I.

A subfield of the EGS with complete coverage in all wave bands, known as the minitest region (MTR), is used in this Letter. The MTR consists of two side-by-side ACS pointings (22 $\left.\mathrm{arcmin}^{2}\right)$ centered at $\alpha=14^{\mathrm{h}} 17^{\mathrm{m}} 52.0^{\mathrm{s}}, \delta=+52^{\circ} 29^{\prime} 3.0^{\prime \prime}$ (see Fig. 1 of Paper I). Postage-stamp images around each galaxy were visually inspected for source blending (chance superpositions or physical pairs), cosmetic problems (CCD edge, cosmic ray, etc.), and missing entries in the photometric catalogs for obvious sources. Of the 101 objects with secure redshifts in the MTR, 30 were dropped, yielding 71 objects with clean measurements. Ten of the dropped objects are confused/blended, 10 are affected by cosmetic problems, and 10 are missing catalog entries (nine of the 10 are missing flux entries in all four IRAC bands because the available IRAC photometry catalog is restricted to sources detected in band 4 $[8 \mu \mathrm{m}])$.

The exclusion of the $10 \mathrm{blended} /$ confused objects, some of which may be physically interacting, does not appear to have introduced a significant bias into our sample. Three of the 10 cases are spectroscopically confirmed to be chance superpositions. No redshift information is available for the neighbor in the remaining seven cases, but their color and morphology distribution is identical to that of the full sample.

Our data come from a wide range of instruments, each with its own complexities. We have therefore relied on the expertise of each team to obtain photometry. The total magnitudes are derived from aperture photometry using apertures of diameter 2-3 times the FWHM of the PSF. The rest-frame absolute magnitude $M_{B}$ and $U-B$ color are derived from the optical photometry using the $K$-correction procedure described in Willmer et al. (2006).

\section{DETECTION LIMITS IN THE CONTEXT OF GALAXY MODELS}

In this section, we examine the AEGIS detection limits in the context of typical galaxy SEDs. BC03 stellar population synthesis models provide a simple conceptual framework. Those containing only stellar emission-a complete treatment of dust extinction or emission from dust, gas, and AGNs-is beyond the scope of this work. For simplicity, we consider two models:

The first is a passive evolution model in which the stars form early, at a redshift $z_{f}=10$, over a very short period, $\sim 10 \mathrm{Myr}$. The stellar mass in the model is tuned such that its $R$-band luminosity is at our detection limit at $z=0.7$. A galaxy with stellar mass of $M_{*}=8 \times 10^{10} M_{\odot}$ is bright enough to be detected in the AEGIS optical R/I and Spitzer mid-IR bands.

Second, we consider a model in which the star formation rate is constant from a redshift of $z_{f}=10$ until it is "observed" at $z_{\mathrm{obs}}=0.7$. As before, the star formation rate is tuned so that the $R$-band luminosity is at our detection threshold at $z=0.7$. The redshift range $z_{f}=10$ to $z_{\mathrm{obs}}=0.7$ corresponds to $6.7 \mathrm{Gyr}$.

Figure 1 shows the SEDs of both models (red: early burst; blue: constant star formation rate), compared to the detection limits of our survey (downward arrows: typically $5 \sigma$ limits; see Paper I). Note that the $24 \mu \mathrm{m}$ Spitzer/MIPS band's detection limit is well above the model stellar SEDs.

\section{SPECTRAL ENERGY DISTRIBUTIONS}

Rest-frame SEDs for 11 galaxies are shown in Figure 2 (left panels). This subsample spans a representative range of optical colors and luminosities. The only two Chandra sources in our sample, most likely AGNs, are shown at the top of the figure. The flux density is normalized to the $R$ band, and detection limits are shown with upward arrows. The effective rest-frame frequency, $\nu_{\text {eff }}$, of each data set is shown by a tick mark at the top of each SED panel. The two BC03 model SEDs described in $\S 3$ are overlaid, normalized to the rest-frame $R$ band: the constant star formation rate model (blue line) and the early burst model (red line). These models do not include any dust absorption/emission. The DEEP2 object ID, redshift, and SED type ( $\$ 4.2)$ are listed in the top right corner of each left panel. The middle panels show true-color images formed by combining the HST ACS images in the $V+I$ bands using the algorithm of Lupton et al. (2003). The right panels show the location of the galaxy in a plot of rest-frame absolute $B$-band magnitude versus rest-frame $U-B$ color ( $r e d$ dot) relative to other AEGIS galaxies in a redshift slice around the target, $z_{\text {targ }} \pm 0.1$.

Table 1 lists the SEDs of the 11 galaxies that are shown in Figure 2. The radio upper limits are listed in Paper I.

\subsection{Notes on Individual Objects}

In this section, we describe the morphology, spectral features, and field crowding of each of the 11 selected objects. No individual galaxy in this subsample of 11 is a VLA 6 or $20 \mathrm{~cm}$ detection. Emission lines are listed when clearly visible by eye in order to convey a sense of the features used for redshift determination. For an emission line to be visible in a typical galaxy within our sample, its equivalent width must be $\gtrsim 4 \AA$.

12007878 is an AGN with a red core and an asymmetric underlying disk. It shows [O II] emission. The Chandra irradiances for this object in the hard/soft bands are (1.7 \pm $0.2) \times 10^{-14} /(3.0 \pm 0.3) \times 10^{-15} \mathrm{ergs} \mathrm{s}^{-1} \mathrm{~cm}^{-2}$.

12007954 is an AGN, with [O II] emission and a bright nuclear source visible in HST ACS. This object is discussed 

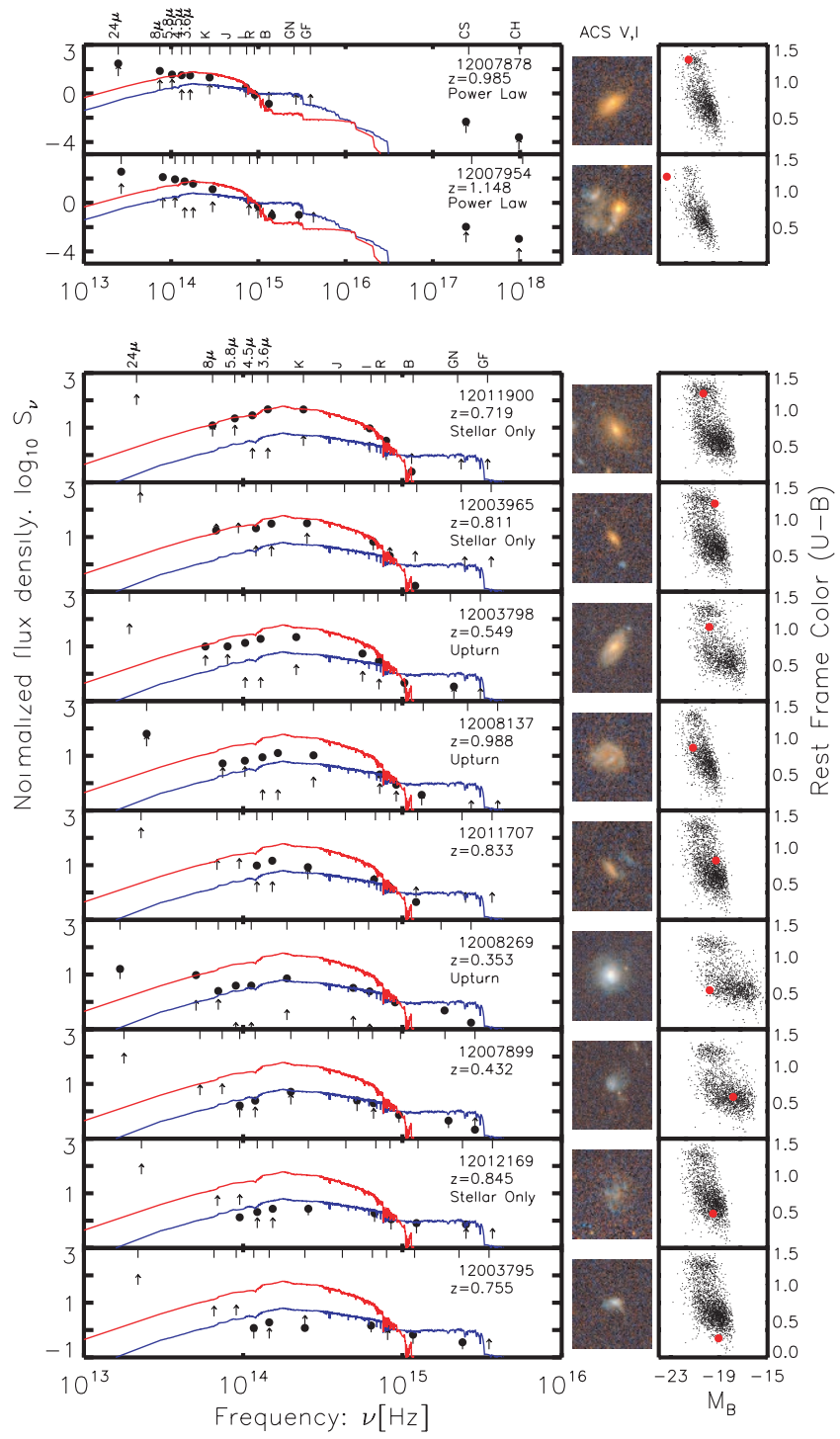

FIG. 2.-Properties of 11 representative galaxies in the MTR that have been hand-picked to ensure minimal neighbor contamination and optical-color diversity. Left: Panchromatic SED (flux-density vs. frequency) of the galaxy compared to simple stellar population models from BC03: early burst (red) and constant star formation rate (blue); see $\S 3$ for details. The model SEDs tend to bracket the near-IR to UV portion of the observed SEDs. The DEEP2 object ID, redshift, and SED type (§ 4.2) are listed in the top right corner of each panel. Middle: "True"-color image based on HST ACS images in the F606W $(V)$ and F814W $(I)$ bands (near right). The images are $50 \mathrm{kpc}$ (comoving) on a side. Right: Rest-frame $U-B$ vs. $M_{B}$ color-magnitude diagram, showing the target galaxy (large red dot) relative to other DEEP2 galaxies whose redshifts lie within $z_{\text {targ }} \pm 0.1$ (small black dots).

by Le Floc'h et al. (2007). The Chandra hard/soft irradiances are $(3.8 \pm 0.2) \times 10^{-14} /(1.3 \pm 0.1) \times 10^{-14} \mathrm{ergs} \mathrm{s}^{-1} \mathrm{~cm}^{-2}$.

12011900 is a red early-type galaxy. The bright IRAC source to the northeast may be a contaminant.

12003965 is a faint red irregular object, with dust lanes. A faint optical companion may contaminate the IRAC fluxes. [O II] is present.

12003798 is a spiral galaxy in the "valley" between red and blue galaxies. This galaxy has an [O III] doublet, [Ne III], and Balmer emission.

12008137 is a spiral galaxy with dust lanes. [O II] emission is seen.
12011707 is a dusty irregular galaxy. Its spectrum shows [O II] and Balmer emission.

12008269 has a very bright core and many distinct parts. The object has strong Balmer, [O III], and [Ne II].

12007899 is a blue irregular object. The spectrum shows strong [O III] and a Balmer emission sequence.

12012169 is a spiral galaxy, with no visible spheroidal component. The [O III], Balmer, and [O II] lines are strong with a hint of weak [Ne III].

12003795 is a very blue irregular object. It shows a sequence of strong emission lines: [O II], [O III], Balmer lines, and the high ionization potential lines $[\mathrm{Ne}$ III] and $[\mathrm{He} \mathrm{I}]$.

\subsection{SED Types}

We classify each of the 71 nonblended SEDs into one of three types. "Stellar" types follow BC03 SEDs; examples of this class are objects 12011900,12012169 , and 12003965 in Figure 2. "IR-excess" types display a mid- to far-IR excess relative to a pure stellar SED (IRAC $8 \mu \mathrm{m}$ upturn and/or MIPS $24 \mu \mathrm{m}$ detection); examples include objects 12003798, 12008137 , and 12008269. The third type, "power law," is characterized by an SED of the form $S_{\nu} \propto \nu^{\alpha}$; the only two such objects in our sample are 12007878 and 12007954 . Note that the AEGIS MIPS $24 \mu \mathrm{m}$ detection limit is relatively bright (Fig. 1) so that some of the stellar SEDs may well have upturns in the far-IR that fall below our detection threshold. Finally, galaxies that do not have detections in IRAC bands 3 or 4 are difficult to classify; these include objects 12001707, 12007899, and 12003795. The SED types include 22 stellar, 31 IR-excess, two power-law, and 16 objects that are missing IRAC fluxes.

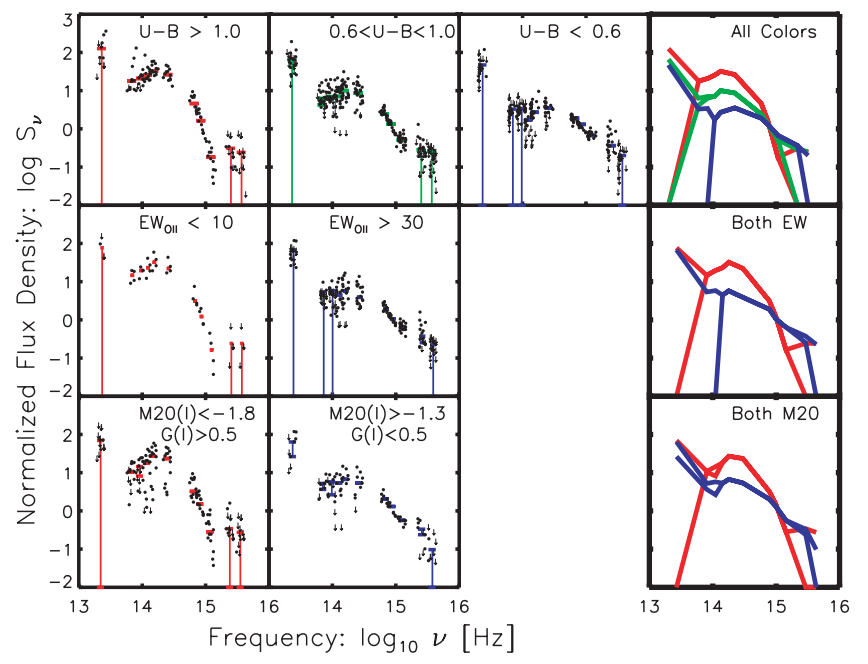

FIG. 3.-Grouped SEDs of the galaxies in our sample in a flux density vs. frequency plot, with the grouping criteria indicated (seven left panels with thin outlines). The small black dots indicate flux densities for individual galaxies, and the colored dots (vertical bars) indicate the median flux density (median range) for that band (see $\S 5$ ). The set of three panels to the right with bold outlines show each group's median SED as a bold colored line, following the same color scheme as the panels to the left. The median panchromatic SED shapes are seen to be generally correlated with the optical properties on which the grouping criteria are based-i.e., red rest-frame $U-B$ color, early-type morphology, and weak [O II] line emission are associated with SEDs that have steep UV to IR slopes (red) while blue $U-B$, late-type morphology, and strong [O II] emission are associated with SEDs that have shallow UV to IR slopes (blue). 
TABLE 1

Selected Spectral Energy Distributions

\begin{tabular}{|c|c|c|c|c|c|c|c|c|c|c|c|c|}
\hline Object & $z^{\mathrm{a}}$ & $\mathrm{GN}^{\mathrm{b}}$ & $\mathrm{GF}^{\mathrm{c}}$ & $B$ & $R$ & $I$ & $K$ & IRAC1 & IRAC2 & IRAC3 & IRAC4 & MIPS24 \\
\hline 12007878 & 0.985 & $\downarrow-1.551$ & $\downarrow-1.551$ & -1.468 & -0.726 & -0.121 & 0.698 & 0.868 & 0.882 & 0.937 & 1.235 & 1.846 \\
\hline $12007954 \ldots \ldots$ & 1.148 & $\downarrow-1.697$ & -1.056 & -1.144 & -0.321 & 0.136 & 1.053 & 1.506 & 1.698 & 1.866 & 2.052 & 2.494 \\
\hline 12011900 & 0.719 & $\downarrow-0.918$ & $\downarrow-0.918$ & -1.002 & 0.131 & 0.584 & 1.275 & 1.278 & 1.059 & 0.948 & 0.691 & $\downarrow 1.441$ \\
\hline $12003965 \ldots \ldots$ & 0.811 & $\downarrow-1.127$ & $\downarrow-1.127$ & -1.809 & -0.713 & -0.197 & 0.478 & 0.463 & 0.294 & $\downarrow 0.112$ & 0.213 & $\downarrow 1.232$ \\
\hline $12003798 \ldots \ldots$ & 0.549 & -1.142 & -0.533 & -0.394 & 0.399 & 0.672 & 1.275 & 1.210 & 1.063 & 0.935 & 0.931 & $\downarrow 1.384$ \\
\hline $12008137 \ldots \ldots$ & 0.988 & $\downarrow-1.498$ & $\downarrow-1.498$ & -0.914 & -0.543 & -0.168 & 0.532 & 0.615 & 0.462 & 0.335 & 0.234 & 1.320 \\
\hline $12011707 \ldots \ldots$ & 0.833 & $\downarrow-1.318$ & $\downarrow-1.318$ & -1.290 & -0.822 & -0.466 & -0.016 & 0.219 & 0.043 & $\downarrow-0.079$ & $\downarrow-0.115$ & $\downarrow 1.041$ \\
\hline $12008269 \ldots$. & 0.353 & -0.293 & 0.154 & 0.449 & 0.850 & 0.979 & 1.325 & 1.062 & 1.061 & 0.866 & 1.446 & 1.673 \\
\hline $12007899 \ldots \ldots$ & 0.432 & -1.204 & -0.873 & -0.665 & -0.236 & -0.131 & 0.180 & -0.143 & -0.321 & $\downarrow 0.094$ & $\downarrow 0.059$ & $\downarrow 1.214$ \\
\hline $12012169 \ldots \ldots$ & 0.845 & $\downarrow-1.390$ & -0.862 & -0.814 & -0.675 & -0.456 & -0.291 & -0.292 & -0.408 & -0.604 & $\downarrow-0.186$ & $\downarrow 0.969$ \\
\hline $12003795 \ldots \ldots$ & 0.755 & $\downarrow-1.384$ & -1.128 & -0.851 & -0.623 & -0.515 & -0.603 & -0.399 & -0.605 & $\downarrow-0.145$ & $\downarrow-0.180$ & $\downarrow 0.975$ \\
\hline
\end{tabular}

${ }^{a}$ Typical uncertainty in redshift is $3.4 \times 10^{-5}$.

${ }^{\mathrm{b}}$ GALEX Near; all flux densities are in $\log (\mu \mathrm{Jy})$. Note that $1 \mu \mathrm{Jy}=10^{-29} \mathrm{ergs} \mathrm{s}^{-1} \mathrm{~Hz}^{-1} \mathrm{~cm}^{-2}$ and $\mathrm{AB}$ magnitudes are defined as $-2.5 \log I_{\nu}-48.6$, where $I_{v}$ is in units of ergs s$~^{-1} \mathrm{~Hz}^{-1} \mathrm{~cm}^{-2}$. An upper limit is denoted by a downward-pointing arrow.

${ }^{\circ}$ GALEX Far.

\section{TRENDS WITH OPTICAL PROPERTIES}

To give the reader an idea of the richness of the AEGIS data, each galaxy is grouped according to the following parameters: $U-B$ color, [O II] equivalent width, and Gini/ $M_{20}$ in the observer-frame $I$ band (Lotz et al. 2004). Gini/ $M_{20}$ is a quantitative measurement of the galaxy's morphology. These groupings are chosen for their broad-stroke ability to create similar groups of objects, even though the parameters are not independent. That is, red galaxies generally have low [O II] emission and early-type morphologies (Bell et al. 2004; Weiner et al. 2005), while blue galaxies generally have strong [O II] emission and later type morphology (Kennicutt 1998).

Figure 3 overplots all 71 SEDs (small black dots in seven left panels) grouped by $U-B$, [O II] line strength, and Gini/ $M_{20}$. The grouping criteria are indicated in the top right of each panel. Colored dots (vertical bars) show the median normalized flux value (range) for each band.

The set of three panels on the right show the median SEDs of each group as bold colored lines (where the branching indicates the "median range," as discussed below). If all galaxies were detected in all bands, the median SED would exist. In reality, most galaxies are not detected in all bands. To take advantage of detection limits, we introduce the median range. The upper end of the range is the median of our measurements but with upper limits replaced with detection limits. The lower end of the range is the median of a list where nondetections are replaced with 0 . If all galaxies are undetected in a given band, then the median range is the pair (median of the normalized detection limits, 0 ).

\section{RESULTS}

The near-IR to UV portions of the SEDs of the galaxies in our sample, typically luminous galaxies at $z \sim 1$, are generally bracketed by the early burst and constant star formation rate model stellar SEDs (Fig. 2). This indicates that few, if any, of the luminous galaxies at these epochs are undergoing significant starbursts. This portion of the SED appears to be closely correlated, at least in an average sense, with the rest-frame UV and optical properties such as $U-B$ color, [O II] emissionline strength, and morphology. These same UV/optical properties do not appear to be good predictors of whether the overall SED follows a pure stellar population model (stellar model), shows a mid- to far-IR excess relative to a pure stellar population model (IR-excess model), or follows a power law (powerlaw model). About $60 \%$ of the galaxies have detectable midto far-IR emission; this is likely thermal emission from interstellar dust. A few percent of the SEDs are of the form $S_{\nu} \propto$ $\nu^{\alpha}$, likely because they are dominated by AGNs.

The authors wish to thank D. C. Koo for conceiving and helping with this Letter and the anonymous referee for valuable comments. N. P. K. and J. M. L. are supported by NASA grant HST-GO-10314.18-A. A. L. C. and J. A. N. are supported by NASA through Hubble Fellowship grants HF-01182.01-A and HF-01182.01-A. The authors wish to recognize and acknowledge the very significant cultural role and reverence that the summit of Mauna Kea has always had within the indigenous Hawaiian community. We are most fortunate to have the opportunity to conduct observations from this mountain.

\section{REFERENCES}

Bell, E. F., et al. 2004, ApJ, 600, L11

Bruzual, G., \& Charlot, S. 2003, MNRAS, 344, 1000 (BC03)

Cooper, M., Newman, J., Madgwick, D. S., Gerke, B. F., Yan, R., \& Davis, M. 2005, ApJ, 634, 833

Dale, D. A., Helou, G., Contursi, A., Silbermann, N. A., \& Kolhatkar, S. 2001, ApJ, 549, 215

Davis, M., et al. 2003, Proc. SPIE, 4834, 161

2007, ApJ, 660, L1 (Paper I)

Giavalisco, M., et al. 2004, ApJ, 600, L93
Kennicutt, R. C., Jr. 1998, ARA\&A, 36, 189

Le Floc'h, E., et al. 2007, ApJ, 660, L65

Lotz, J., Primack, J., \& Madau, P. 2004, AJ, 128, 163

Lupton, R., Blanton, M., Fekete, G., Hogg, D. W., O’Mullane, W., Szalay, A., \& Wherry, N. 2003, preprint (astro-ph/0312483)

Mushotzky, R. F., Marshall, F. E., Boldt, E. A., Holt, S. S., \& Serlemitsos, P. J. 1980, ApJ, 235, 377

Weiner, B. J., et al. 2005, ApJ, 620, 595

Willmer, C. N. A., et al. 2006, ApJ, in press (astro-ph/0506041) 